\title{
p300-Dependent Chromatin Remodelling Regulates Multiple Liver Functions
}

Leila Valanejad and Nikolai Timchenko*

Division of Pediatric General and Thoracic Surgery, Cincinnati Children's Hospital Medical Center, 3333 Burnet Avenue, Cincinnati, OH, USA

\begin{abstract}
Liver is a one of the largest tissues that performs a variety of complex functions which support body homeostasis including detoxification and providing essential molecules to the blood. Disorganization of liver functions is one of the main characteristics of several severe diseases including liver cancer and non-alcoholic fatty liver disease (NAFLD). Understanding the mechanisms which control functions of the healthy liver is highly important for development of approaches for treatments of liver diseases. Although the role of chromatin remodeling in liver biology has been documented by many reports, the precise mechanisms of this regulation are not well understood. We have recently determined these mechanisms using transgenic mice which express a dominant negative p300 molecule, dnp300 mice. This animal model is an excellent tool for the examination of the role of p300 since activities of the endogenous p300 are inhibited in these mice. An examination of global changes in expression of genes in livers of dnp300 mice revealed that p300 regulates multiple pathways. These pathways include chromatin remodeling, DNA damage, fatty liver, oncogenes, apoptosis, cell cycle and translation. One of the key specific pathways of p300-dependent regulation of liver functions is the co-operation of p300 with C/EBP family proteins. Our long term studies of C/ EBPa-p300 complexes in biological processes of the liver included investigations in wild type mice and in genetically modified animal models with elevated or reduced amounts of C/EBPa-p300 complexes. Examination of these animal models elucidated mechanisms by which C/EBPa-p300 complexes are involved in development of NAFLD and in regulation of liver proliferation/regeneration and liver injury. This review summarizes the knowledge obtained in these genetically-modified animal models with altered chromatin structure.
\end{abstract}

Keywords: p300; Chromatin remodelling; Liver proliferation; NAFLD; C/EBP

\section{Introduction}

Biology of the liver and liver functions is regulated by extremely complex communications of different signaling pathways and layers of gene expression. Disorders of these communications lead to development of liver diseases such as hepatocellular carcinoma, HCC [1-4]. It has been also shown that biological pathways are changed in aged liver and that liver proliferation after surgery is impaired in aging mice [5]. Numerous studies from many groups found that key genes involved in liver function include CCAAT enhancer-binding protein (C/EBP) family, $\mathrm{Rb}$ family, histone deacetylases 1 (HDAC1), p300 [3,5-7] and RNA binding protein CUGBP1 [7]. A member of the $\mathrm{C} / \mathrm{EBP}$ family, $\mathrm{C} / \mathrm{EBP} \alpha$, inhibits liver proliferation and protects the liver from the development of tumors by direct interactions with cell cycle proteins and with chromatin remodelling proteins $[3,8-10]$. Our examination of effects of age on biology of the liver showed that aging phosphorylates $\mathrm{C} / \mathrm{EBP} \alpha$ at $\mathrm{S} 193$ resulting in inhibition of liver proliferation by promoting the formation of $\mathrm{HDAC} 1$ and $\mathrm{C} / \mathrm{EBPa}$ complexes [5,7-10]. Generation of two knockin mouse models with a constitutively active C/EBPa-S193D and with C/EBPa-S193A mutant revealed that phospho-mimicking mutation (S193D) inhibits liver proliferation [8]; while mutation of $S 193$ to Ala resulted in an increase of liver proliferation after partial hepatectomy and to a failure to stop liver regeneration [3]. Similar to C/EBPa, another C/EBP family member, $\mathrm{C} / \mathrm{EBP} \beta$, is a key regulator of liver functions such as liver proliferation and differentiation. Although $\mathrm{C} / \mathrm{EBP} \beta$ is an intronless gene, a single $\mathrm{C} / \mathrm{EBP} \beta$ mRNA contains three-in-frame AUG codons and translates three isoforms, full-length $(\mathrm{C} / \mathrm{EBP} \beta-\mathrm{FL})$, liver activating protein $(\mathrm{C} /$ EBP $\beta$-LAP), and liver inhibitory protein (C/EBP $\beta$-LIP) [11]. All three isoforms contain DNA binding domain, but possess differential activities because of differences in the activation domains. Among these isoforms, C/EBP $\beta$-LIP completely lacks activation domains and works as a dominant negative molecule. It has been shown that C/EBP $\beta$-LIP directly interacts with $\mathrm{Rb}$ and disrupts $\mathrm{E} 2 \mathrm{~F}-\mathrm{Rb}$ complexes leading to de-repression of E2F-dependent promoters and to proliferation [12]. Among several families of chromatin remodeling proteins, histone acetyl-transferase (HAT) p300 has been shown to be a critical regulator of liver biology. Alterations of levels of p300 have been documented for several liver diseases. For example, it was found that p300 is highly expressed in HCC $[13,14]$. Previous studies have also shown that the inhibition of HAT activity of p300 decreases hepatic steatosis [15]. In agreement with these observations, the deletion of $\mathrm{CH} 1$ domain of p300 resulted in resistance of mice to high fat diet-mediated elevation of triglycerides [16]. In addition to these functions of $\mathrm{p} 300$, generation of mice with hepatic-specific deletion of $\mathrm{p} 300$ and mice with knockin p300-G422S revealed that p300 also regulates glycogen synthesis through maintaining basal gluconeogenesis $[17,18]$. Despite these important observations, very little is known about mechanisms by which p300 regulates liver functions.

Chromatin Structure, Liver Morphology and Liver Functions are Altered in Three Animal Models with Increased or Reduced Amounts of C/EBPa-P300 Complexes

Histone acetyl transferase p300 regulates chromatin structure through interactions with many transcription factors, TFs [19]. Our lab is investigating the role of C/EBP-p300 family complexes on the

*Corresponding author: Nikolai Timchenko, Division of Pediatric General and Thoracic Surgery, Cincinnati Children's Hospital Medical Center, 3333 Burnet Avenue, Cincinnati, OH, USA, Tel: 513-636-0129; E-mail: Nikolai.Timchenko@ cchmc.org

Received December 03, 2015; Accepted January 23, 2016; Published January 25, 2016

Citation: Valanejad L, Timchenko N (2016) p300-Dependent Chromatin Remodelling Regulates Multiple Liver Functions Single Cell Biol 5: 126. doi:10.4172/21689431.1000126

Copyright: (c) 2016 Valanejad L, et al. This is an open-access article distributed under the terms of the Creative Commons Attribution License, which permits unrestricted use, distribution, and reproduction in any medium, provided the original author and source are credited. 
epigenetic regulation of liver biology. Initially, the primary focus of our studies was $\mathrm{C} / \mathrm{EBP} \alpha$ and its interaction with chromatin remodeling proteins. Although C/EBPa-p300 complexes have been implicated in regulation of liver biology, there was no genetic evidence showing the co-operation of these molecules in biological process of the liver. The interaction of C/EBPa with p300 is controlled by cdk4-dependent phosphorylation of C/EBPa at Ser193 [2,3,8]. As we mentioned above, our lab generated three genetically modified animal models in which amounts of C/EBPa-p300 complexes are significantly increased: C/ EBPa-S193D mice (S193D) or dramatically reduced: C/EBPa-S193A mice (S193A) and dnp300 mice. Investigation of our first model, S193D, showed alterations of chromatin structure of the liver at young age that is typically observed in livers of old mice [9]. These changes included enlarged hepatocytes and abundant foci-liked structures of nuclei which contained markers of heterochromatin. Initial studies of this model revealed that this change of chromatin structure is associated with increase of complexes of $\mathrm{C} / \mathrm{EBP} \alpha$ with another chromatin remodeling protein, histone deacetylase-1, HDAC1 [9]; however later investigation found that amounts of C/EBPa-p300 complexes are also elevated in the S193D mice $[2,3]$. Consistent with the role of $\mathrm{p} 300$ and C/EBPa, chromatin structure is changed in livers of two other animal models S193A and dnp300 mice $[1,3]$. We next investigated the consequences of the alterations of $\mathrm{C} / \mathrm{EBP} \alpha-\mathrm{p} 300$-dependent chromatin structure. Examination of blood parameters in S193A versus S193D mice revealed that the C/EBP $\alpha-p 300$ complexes are involved in the regulation of liver damage. While the overexpression of C/EBP $\alpha-p 300$ complexes in S193D mice causes elevation of alanine amino transaminase and aspartate amino transaminase (ALT/AST) in serum [8], S193A mice with low amounts of these complexes have reduced ALT/AST levels [3]. These alterations showed that $\mathrm{C} / \mathrm{EBP} \alpha-\mathrm{p} 300$ complexes are involved in the regulation of the liver damage. In addition to that, levels of triglycerides (TG) and very low density lipoproteins (VLDL) are increased in the blood of S193D mice, but are reduced in the S193A mice. We also found that levels of glucose in the serum are under the control of $\mathrm{C} /$ $\mathrm{EBP} \alpha-\mathrm{p} 300$ complexes and that glucose is reduced in the S193A mice. Chromatin immunoprecipitation, QRT-PCR and western blotting revealed that low levels of glucose in S193A mice are mediated by the reduction of the C/EBP $\alpha-p 300$ complexes on the promoters of enzymes of glucose synthesis PEPCK and G6Pase leading to subsequent decrease of corresponding mRNAs and proteins [3]. While livers of S193A and S193D mice showed mainly C/EBPa-p300-dependent alterations, dnp300 mice have several additional alterations of blood parameters because p300 interacts with many other transcription factors. These additional alterations include a dramatic reduction of creatinine and gamma glutamyltransferase [1]. Taken together, the morphological and biochemical analyses of animal models with altered C/EBPa-p300 complexes and chromatin structures revealed an essential role of chromatin structure and these complexes in liver biology and liver functions.

\section{Role of p300-Dependent Chromatin Remodelling in Development of NAFLD}

NAFLD is one of the most devastating diseases that affect many patients $[20,21]$. The development of NAFLD includes several steps. The first step of NAFLD is hepatic steatosis which is characterized by accumulation of TG's in cytoplasm of hepatocytes. Hepatic steatosis can further progress to non-alcoholic steatohepatitis (NASH) which is characterized by more severe liver damage. NASH further progresses to cirrhosis and to hepatocellular carcinoma [20,21]. Dietary based animal models of non-alcoholic fatty liver disease provided significant information regarding liver damage and metabolic pathways of hepatic steatosis [21]. Despite numerous studies of hepatic steatosis in these animal models of non-alcoholic fatty liver disease, molecular mechanisms which cause hepatic steatosis are not well understood. Examination of fatty liver characteristics (fat droplets, Oil-Red-O staining and liver TG levels) revealed that S193D mice develop hepatic steatosis at age of 2 months [2]; while S193A and dnp300 mice do not have hepatic steatosis at this age and do not develop it even at age 1820 months [1,3]. Mouse models with reduced C/EBP $\alpha-p 300$ complexes have also reduced levels of TG; while S193D mice have elevated levels of serum TG. We have elucidated precise mechanisms by which $\mathrm{C} /$ EBPa-p300 complexes increase levels of TG. These mechanisms include binding of C/EBPa-p300 complexes to promoters of 5 enzymes of TG synthesis and activation of their expression through chromatin remodeling pathway [2]. S193D and double S193D-dnp300 mice were used for examination of development of hepatic steatosis under conditions of high fat diet (HFD) which is the most common source of NAFLD in humans. These studies revealed that S193D mice develop hepatic steatosis much faster than WT mice and that the crossing of S193D mice with dnp300 mice reduces levels of C/EBPa-p300 complexes and completely inhibit development of hepatic steatosis. In agreement with these observations, the inhibition of $\mathrm{p} 300$ by siRNA in mice with existing hepatic steatosis significantly reduces the hepatic steatosis [2]. The results with our three animal models are consistent with observations published by Shao's group [22]. In this paper, the authors knocked down C/EBPa in livers of WT and in livers of diabetic $\mathrm{db} / \mathrm{db}$ mice by adenoviral delivery of siRNA and found that the inhibition of $\mathrm{C} / \mathrm{EBP} \alpha$ in $\mathrm{db} / \mathrm{db}$ mice leads to the reduced hepatic glucose production and to decrease of lipid accumulation and triglycerides levels through the reduction of lipogenic gene expression [22]. Although the authors did not examine a precise mechanism by which inhibition of $\mathrm{C} / \mathrm{EBP} \alpha$ causes these changes, it is very likely that, similar to data with our $\mathrm{C} /$ EBPa-S193A mice [3], the C/EBPa-p300 complexes were reduced on the promoters of the PEPCK, G6Pase and hepatic lipogenic genes in livers of $\mathrm{db} / \mathrm{db}$ mice leading to the reduction of corresponding mRNAs and proteins. Taking together our studies of genetically modified animal models with different levels of $\mathrm{C} / \mathrm{EBP} \alpha-\mathrm{p} 300$ complexes and results with liver specific inhibition of $\mathrm{C} / \mathrm{EBPa}$ [22], it is clear that the $\mathrm{C} / \mathrm{EBPa}-\mathrm{p} 300$ pathway plays a critical role in regulation of gluconeogenesis and in development of NAFLD.

\section{p300-Dependent Pathways Regulate Liver Regeneration after Surgery}

The liver is a unique tissue which is able to regenerate after massive surgical resections [23]. Although mechanisms of liver regeneration after surgery are very well investigated [24], the role of epigenetic control and chromatin remodeling in this process is not understood. The generation of the three animal models with the alterations of chromatin structure allowed us to directly examine the role of chromatin structure in liver regeneration. Therefore, we have applied a $2 / 3$ partial hepatectomy model $(\mathrm{PH})$ of liver regeneration. In this approach, $70 \%$ of the liver is removed and the remaining portion starts proliferation and restores to the original size. The initial studies with $\mathrm{C} / \mathrm{EBP} \alpha-\mathrm{S} 193 \mathrm{D}$ mice clearly demonstrated that overexpression of $\mathrm{C} / \mathrm{EBPa}-\mathrm{p} 300$ complexes is in fact a growth inhibitory event. Several approaches including measuring DNA replication, mitotic figures and cell cycle proteins revealed that liver proliferation is almost completely inhibited in these mice [8]. On the contrary, S193A livers with low levels of C/EBPa-p300 complexes enter cell cycle early, proliferate faster and do not stop regeneration at the time when WT livers do [3]. The PH studies in S193A mice elucidated mechanisms by which liver stops proliferation after surgery and showed that proper amounts of C/EBP $\alpha-\mathrm{p} 300$ complexes are required for the termination of liver regeneration. In addition to that, 
this work revealed a complexity of chromatin remodeling regulation and showed that histone deacetylase-1, HDAC1, competes with p300 for the interactions with $\mathrm{C} / \mathrm{EBPa}$ and it is involved in the regulation of p300-dependent liver functions via this competition [3,8]. Our recent studies of dnp300 mice made a significant contribution to further understanding of the role of p300-dependent chromatin remodeling in liver regeneration. Consistent with observations in S193A mice which have low levels of C/EBPa-p300 complexes, the inhibition of p300 by dnp300 molecule dramatically increases liver regeneration after surgery and destroys the mechanisms of termination of liver proliferation after $\mathrm{PH}$ leading to the increase of liver proliferation after $\mathrm{PH}[1]$.

\section{Role of p300 in Liver Injury and Recovery after Injury}

The liver is the main organ which performs detoxification making it the primary target of chemically induced injury. As the result of this injury, the liver starts proliferating which further leads to the development of several diseases including fibrosis, cirrhosis and hepatocellular carcinoma. To determine the role of p300 and p300-C/ $\mathrm{EBP} \alpha$ complexes in liver injury after chemical exposure, we performed a set of studies in which WT, S193D and dnp300 mice were treated with carbon tetrachloride $\left(\mathrm{CCl}_{4}\right)$ and liver injury and recovery were examined. These studies revealed that animals overexpressing C/EBPa-p300 complexes (S193D mice) have increased rate of proliferation and are more sensitive to development of $\mathrm{CCl}_{4}$-mediated fibrosis [25]. This sensitivity is associated with alterations in expression of key regulators of liver biology such as TERT1, FXR and C/EBP $\beta$ [25]. On the contrary to mice overexpressing $\mathrm{p} 300-\mathrm{C} / \mathrm{EBPa}$ complexes, mice with inhibited p300 and p300-C/EBPa complexes have blocked the response of the liver to $\mathrm{CCl}_{4}$-mediated injury, but have elevated liver proliferation [1]. Searching for the underlying mechanisms of the resistance of dnp300 mice to liver injury, we found that the inhibition of p300 affects several additional pathways of liver biology. The most important pathways include changes on the levels of post-translational modifications which lead to accumulation of translational repression complexes of RNA binding protein, CUGBP1 with inactive form of translation initiation factor eIF2, and subsequent translational inhibition of p53. It is interesting to note that previous studies showed that p53 recruits p300 to the promoter of tumor suppressor Foxo3 gene and increases expression of Foxo3 in quiescent livers and that this regulation is disrupted after $\mathrm{PH}$ leading to the loss of Foxo3 expression during the proliferative stage of liver regeneration [26]. Our studies of livers of dnp300 mice without challenges demonstrated that a portion of hepatocytes proliferates and they are on the stages of DNA replication and mitosis [1]. Moreover, livers of dnp300 mice have significantly increased proliferation after $\mathrm{PH}$ and $\mathrm{CCl}_{4}$ treatments. Given the reduction of p300 activity and reduction of p53 in livers of dnp300 mice, it is likely that a possible inhibition of Foxo3 is involved in the basic increase of liver proliferation and in acceleration of liver proliferation of dnp300 mice after $\mathrm{PH}$ and $\mathrm{CCl}_{4}$ treatments.

\section{Conclusions and Future Directions}

Alterations of chromatin structure in our three animal models significantly change expression of genes at different levels. One additional alteration in gene expression in our animal models is the accumulation of a truncated C/EBP $\beta$-isoform, C/EBP $\beta$-LIP. Although we have not examined directly the role of this isoform in increased proliferation observed after $\mathrm{PH}$ in $\mathrm{C} / \mathrm{EBP} \alpha-\mathrm{S} 193 \mathrm{~A}$ and dnp300 mice, it is likely that the elevation of C/EBP $\beta$-LIP contributes to the increase of liver proliferation in these animal models. Figure 1 summarizes results presented in our recent paper [1] and in our other publications of long-

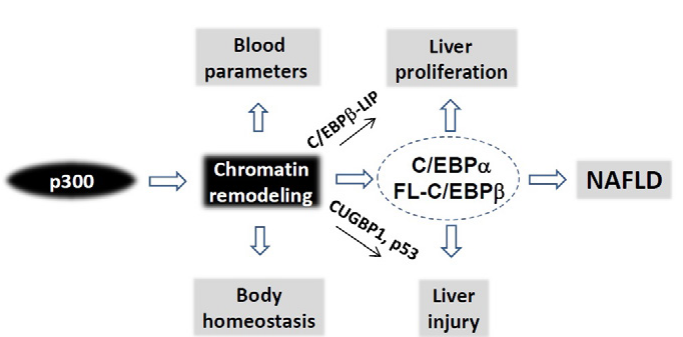

Figure 1: p300 controls body homeostasis and multiple liver functions as well as development of NAFLD through multiple pathways.

term studies of the role of $\mathrm{p} 300$-dependent chromatin remodeling in liver biology $[1-3,8,9,25]$. These studies demonstrated that $\mathrm{p} 300$ controls development of NAFLD and is a key protein which regulates liver response to surgery and injury. p300-dependent molecular pathways include C/EBPa, full-length (FL) C/EBP $\beta$, C/EBP $\beta$-LIP, CUGBP1 and p53 signaling. It is important to note that C/EBPa and FL-C/EBP- $\beta$ proteins can form heterodimers and regulate expression of genes as triple complexes with p300. Identification of p300-C/EBP pathways as critical regulators of liver injury/proliferation and NAFLD generated a good background for examination if modulations of these pathways might correct/prevent development of liver diseases such as liver cancer and NAFLD. Particularly, we are currently investigating if the inhibition of C/EBPa-p300 pathway might protect/reverse development of NAFLD and correct liver functions. Because C/EBPa-p300 complexes are critical for the termination of liver proliferation [3], it would be also important to develop approaches to support these complexes and potentially prevent/treat liver cancer.

\section{Acknowledgements}

This work is supported NIH grants R01DK102597 and R01CA159942 and by Internal Development Funds from CCHMC (NT).

\section{References}

1. Breaux M, Lewis $K$, Valanejad L, lakova $P$, Chen $C$, et al. (2015) p300 regulates liver functions by controlling $\mathrm{p} 53$ and C/EBP family proteins through multiple signaling pathways. Mol Cell Biol 35: 3005-3016.

2. Jin J, lakova P, Breaux M, Sullivan E, Jawanmardi N, et al. (2013) Increased expression of enzymes of triglyceride synthesis is essential for the development of hepatic steatosis. Cell Rep 3: 831-843.

3. Jin J, Hong IH, Lewis K, lakova P, Breaux M, et al. (2015) Cooperation of C/EBP family proteins and chromatin remodeling proteins is essential for termination of liver regeneration. Hepatology 61: 315-325.

4. Oishi N, Yamashita T, Kaneko S (2014) Molecular biology of liver cancer stem cells. Liver Cancer 3: 71-84.

5. Timchenko NA (2009) Aging and liver regeneration. Trends Endocrinol Metab 20: 171-176.

6. Timchenko NA, Lewis K (2015) Elimination of Tumor Suppressor Proteins during Liver Carcinogenesis. Cancer Studies and Molecular Medicine 1: 27-38.

7. Jones K, Timchenko L, Timchenko NA (2012) The role of CUGBP1 in agedependent changes of liver functions. Ageing Res Rev 11: 442-449.

8. Wang GL,Shi X, Haefliger S, Jin J, Major A, et al. (2010) Elimination of C/ EBPalpha through the ubiquitin-proteasome system promotes the development of liver cancer in mice. J Clin Invest 120: 2549-2562.

9. Jin J, Wang GL, lakova P, Shi X, Haefliger S, et al. (2010) Epigenetic changes play critical role in age-associated dysfunctions of the liver. Aging Cell 9: 895910.

10. lakova $P$, Awad SS, Timchenko NA (2003) Aging reduces proliferative capacities of liver by switching pathways of C/EBPalpha growth arrest. Cell 113: 495-506.

11. Timchenko NA, Wang GL, Timchenko LT (2005) RNA CUG-binding protein 1 
Citation: Valanejad L, Timchenko N (2016) p300-Dependent Chromatin Remodelling Regulates Multiple Liver Functions Single Cell Biol 5: 126. doi:10.4172/2168-9431.1000126

increases translation of 20-kDa isoform of CCAAT/enhancer-binding protein beta by interacting with the alpha and beta subunits of eukaryotic initiation translation factor 2. J Biol Chem 280: 20549-20557.

12. Orellana D, Liu X, Wang GL, Jin J, lakova $P$, et al. (2010) Calmodulin controls liver proliferation via interactions with C/EBPbeta-LAP and C/EBPbeta-LIP. J Biol Chem 285: 23444-23456.

13. Yokomizo C, Yamaguchi K, Itoh Y, Nishimura T, Umemura A, et al. (2011) High expression of p300 in HCC predicts shortened overall survival in association with enhanced epithelial mesenchymal transition in HCC cells. Cancer Letters 310: 140-147.

14. Li M, Luo RZ, Chen JW, Cao Y, Lu JB, et al. (2011) High expression of transcriptional coactivator p300 correlates with aggressive features and poor prognosis of hepatocellular carcinoma. J Transl Med 9: 5 .

15. Bricambert J, Miranda J, Benhamed F, Girard J, Postic C, et al. (2010) Saltinducible kinase 2 links transcriptional coactivator p300 phosphorylation to the prevention of ChREBP-dependent hepatic steatosis in mice. J Clin Invest 120: 4316-4331.

16. Bedford DC, Kasper LH, Wang R, Chang Y, Green DR, et al. (2011) Disrupting the $\mathrm{CH} 1$ domain structure in the acetyltransferases CBP and $\mathrm{p} 300$ results in lean mice with increased metabolic control. Cell Metab 14: 219-230.

17. He L, Cao J, Meng S, Ma A, Radovick S, et al. (2013) Activation of basa gluconeogenesis by coactivator p300 maintains hepatic glycogen storage. Mol Endocrinol 27: 1322-1332.

18. He L, Naik K, Meng S, Cao J, Sidhaye AR, et al. (2012) Transcriptional co- activator p300 maintains basal hepatic gluconeogenesis. J Biol Chem 287 32069-32077.

19. Liu Y, Wang L, Han R, Beier UH, Akimova T, et al. (2014) Two histone/protein acetyltransferases, CBP and p300, are indispensable for Foxp3+ T-regulatory cell development and function. Mol Cell Biol 34: 3993-4007.

20. Cohen JC, Horton JD, Hobbs HH (2011) Human fatty liver disease: old questions and new insights. Science 332: 1519-1523.

21. Hebbard L, George J (2011) Animal models of nonalcoholic fatty liver disease. Nat Rev Gastroenterol Hepatol 8: 35-44.

22. Qiao L, MacLean PS, You H, Schaack J, Shao J (2006) Knocking down liver CCAAT/Enhancer-Binding Protein alpha by adenovirus-transduced silent interfering ribonucleic acid improves hepatic gluconeogenesis and lipid homeostasis in db/db mice. Endocrinology 147: 3060-3069.

23. Michalopoulos GK (2014) Advances in liver regeneration. Expert Rev Gastroenterol Hepatol 8: 897-907.

24. Michalopoulos GK (2013) Principles of liver regeneration and growth homeostasis. Compr Physiol 3: 485-513.

25. Hong I, Lewis K, lakova P, Jin J, Sullivan E, et al. (2014) The age-associated change of C/EBP family proteins causes severe liver injury and acceleration of liver proliferation after CCl4-treatments. J Biol Chem 298: 1106-1118.

26. Kurinna S, Stratton SA, Tsai WW, Akdemir KC, Gu W, et al. (2010) Direct activation of forkhead box $\mathrm{O} 3$ by tumor suppressors p53 and p73 is disrupted during liver regeneration in mice. Hepatology 52: 1023-1032. 